It is clear from this theorem that the Cartan-Goursat calculus of alternating forms* can be developed in Hausdorff spaces with Banach coordinates.

In conclusion we note that Theorem 4 continues to hold if the numerically valued form $\omega$ is replaced by a form $\omega$ with values in a Banach space.

California Institute of Technology

\title{
ON A THEOREM OF ENGEL $\dagger$
}

\section{BY MAX ZORN}

1. Introduction. The theorem of Engel which we intend to study in this paper deals with Lie algebras where an identity $(a(a(a \cdots(a b)) \cdots))=0$ holds for arbitrary elements $a$ and $b$. Under various assumptions it has been shown that in this case all products with sufficiently many factors vanish.

The latest result in this direction was a proof, $\ddagger$ found first by van Kampen, which holds for finite Lie algebras over any field of characterstic zero. The method is rational, but it involves the theory of associative algebras and the theory of traces. Another proof of equal generality, with less accent on the theory of traces, has recently been sketched by the writer. $\$$

The new proof to be offered in the present paper dispenses with every apparatus of matrices, traces, and associative systems. It does not presuppose any knowledge about Lie systems. The material advantage of our direct method is the fact that no reference field is required, and that the question of characteristics never enters the discussion.

2. Definitions. Definition 1. A system L of elements $a, b, \ldots$ is called a Lie ring (with respect to a commutative ring $\mathrm{P}$ of

* E. Goursat, Leçons sur le Problème de Pfaff, 1922; E. Cartan, Leçons sur les Invariants Intégraux, 1922; E. Kahler, Einfïhrung in die Theorie der Systeme von Differentialgleichungen, 1934.

$\dagger$ Substituted for another paper, which was presented to the Society, June 18, 1936. See the last footnote on this page.

$\ddagger$ See N. Jacobson, Rational methods in the theory of Lie algebras, Annals of Mathematics, vol. 36, p. 875.

$\S$ See this Bulletin, Abstract 42-7-266. (Erroneously the theorem in question is there attributed to Lie.) 
scalars $\rho)$ if addition, $a+b$, multiplication, ab, and scalar multiplication, $a \rho=\rho a$, are defined such that (i) the addition defines $a$ group, (ii) the multiplication is distributive, (iii) scalar multiplication is both distributive and associative, (iv) $a a=0$, which implies $a b+b a=0$, (v) $a(b c)+b(c a)+c(a b)=0$ (Jacobi's identity).

Without loss of generality we assume that $\mathrm{P}$ contains \pm 1 .

Definition 2. A subset $S \subset L$ is a subring if it is closed under the operations of Definition 1.

DEFINITION 3. The subring generated from the elements $a, b, \cdots$ and the subsets $A, B, \cdots$ of $L$ is denoted by $\langle a, b, \cdots, A, B, \cdots\rangle$.

Definition 4. If $A_{i}$ are subsets $\subset L, b$ an element $\epsilon L$, then $A_{1}+A_{2}$ is the set of all elements $a_{1}+a_{2}$, where $a_{i} \in A ; A_{1} A_{2}$ the set of all products $a_{1} a_{2} ; A_{1} b$ the set of all $a_{1} b ; \mathrm{P} b$ the set of all scalar multiples $\rho b$.

In this terminology we may announce, for example, the following theorem.

THEOREM 1. If $S$ is a subring and if $S b \subset S$, then $\langle S, b\rangle=S+\mathrm{P} b$.

Since multiplication is in general not associative, the position of parentheses is relevant. For the particular purposes of this paper the following conventions are useful.

Definition 5.

$$
\begin{aligned}
a_{1} a_{2} \cdots a_{n-1} a_{n} & =a_{1}\left(a_{2} \cdots a_{n}\right)=\left(a_{1}\left(a_{2}\left(\cdots\left(a_{n-1} a_{n}\right)\right) \cdots\right)\right), \\
a^{0} b & =b, a^{n} b=a\left(a^{n-1} b\right)=(a(a(\cdots(a(a b))))),
\end{aligned}
$$

and correspondingly $A B=B, A^{n} B=A\left(A^{n-1} B\right)$, and so forth.

Because of $a a=0$, we may very well abandon the usual definition of $a^{2}, \cdots$, so that $a^{n}$ alone does not denote an element of the ring.

Addition and multiplication of subsets satisfy the distributive law

$$
(S+T)(M+N) \subset S M+S N+T M+T N .
$$

Iterated application of this distributivity inclusion yields the following result. 
ThEOREM 2. The set $(S+T)^{n} L$ is contained in a sum of products of type

$$
T^{f_{0} S^{e_{1}}} \cdots S^{e_{k}} T^{f_{k}} L
$$

where $e_{i}>0$ for $i \leqq k, f_{i} \geqq 0, \sum e_{i}+\sum f_{i}=n$.

Jacobi's identity, which takes now the form $a b c+b c a+c a b=0$, furnishes the following lemma.

LEMmA 1. If $S T \subset S$, and $M=-1 \cdot M$, then $T S M \subset S T M+S M$.

This shows that a product like $T S T T \cdots T S \cdots S S T L$ is contained in the sum of two others, such that both still contain the same number of factors $S$, the first has $S T$ instead of $T S$, and the second has one factor $T$ less. From this we obtain the following lemma.

LEMMA 2. If $S T \subset S$, then $T^{f_{0}} S^{e_{1}} \ldots S^{e_{k}} T^{f_{k}} L \subset S^{\Sigma l_{i}} L$.

Definition 6. An element a (a subset $A$ ) is nilpotent if for a suitable exponent $a^{n} L=0$ (respectively, $\left.A^{n} L=0\right)$.

Condition $(1$, iii) implies that if an element $t$ is nilpotent, then the set $T=\mathrm{P} t$ is nilpotent.

\section{Proof of Engel's Theorem.}

Lемма 3. If a proper subring $S$ is nilpotent, $S^{8} L=0$, then there exists an element $t$ which is not contained in $S$ and satisfies $S t \subset S$.

Indeed, since $S^{s} L=0$ there must be a smallest exponent $m$ such that $S^{m+1} L=S\left(S^{m} L\right) \subset S$. The element $t$ may consequently be taken as an arbitrary element of $S^{m} L$ which is not contained in $S$. Such elements do exist, because $S$ is a proper subring and $m \geqq 0$.

If we denote $\mathrm{P} t$ by $T$, we have $S T \subset S$ and this (see Theorem 1) makes $S+T$ a ring which contains $S$ as a proper subring.

LEмma 4. If $S$ and $T$ are nilpotent, and if $S T \subset S$, then $S+T$ is nilpotent.

We shall see that if $S^{s} L=T^{t} L=0$, this nilpotency is exhibited by $(S+T)^{s t} L=0$. On account of Theorem 2 , we have only to prove that 
(2) $\quad T^{f_{0}} S^{e_{1}} \cdots S^{e_{k}} T^{f_{k}} L=0, \quad$ if $\quad \sum_{1}^{k} e_{i}+\sum_{0}^{k} f_{i} \geqq s t$.

Since the condition $S T \subset S$ for (1) is fulfilled, (2) is certainly true if $\sum e_{i} \geqq s$. It is also trivially true if one $f_{i}$ should be $\geqq t$. In any other case we should have at most $s$ exponents $f_{i}$; their sum would be at most $(t-1) s$, and the total sum $\sum e_{i}+\sum f_{i}$ would be smaller than st. Hence there are no such cases, and Lemma 4 is proved. We are now ready to prove Engel's Theorem in the following general form.

ENGEL's THEOREM. If the elements of a subring $R$ are nilpotent, and if in each system of subrings $<R$ there is at least one which is maximal in the system, then $R$ is nilpotent.

For let $S$ be a maximal nilpotent proper subring of $R$. Lemma 3 shows that it is contained (properly) in a ring $S+T \subset R$, where $S T$ с $S ; T=\mathrm{P} t$ is nilpotent. Lemma 4 makes $S+T$ nilpotent, and because it is really greater than $S$ it must be the improper subring $R$. Hence $R$ is nilpotent.

University of California at Los Angeles 